archives-ouvertes

\title{
Magnetic filtration of phase separating ferrofluids: from basic concepts to microfluidic device
}

\author{
P Kuzhir, C Magnet, H Ezzaier, A Zubarev, Georges Bossis
}

\section{To cite this version:}

P Kuzhir, C Magnet, H Ezzaier, A Zubarev, Georges Bossis. Magnetic filtration of phase separating ferrofluids: from basic concepts to microfluidic device. Journal of Magnetism and Magnetic Materials, Elsevier, 2017, 431, pp.84. 10.1016/j.jmmm.2016.08.054 . hal-01356271

\section{HAL Id: hal-01356271 \\ https: / hal.archives-ouvertes.fr/hal-01356271}

Submitted on 25 Aug 2016

HAL is a multi-disciplinary open access archive for the deposit and dissemination of scientific research documents, whether they are published or not. The documents may come from teaching and research institutions in France or abroad, or from public or private research centers.
L'archive ouverte pluridisciplinaire HAL, est destinée au dépôt et à la diffusion de documents scientifiques de niveau recherche, publiés ou non, émanant des établissements d'enseignement et de recherche français ou étrangers, des laboratoires publics ou privés. 


\title{
Magnetic filtration of phase separating ferrofluids: from basic concepts to microfluidic
} device

P. Kuzhir ${ }^{1 *}$, C. Magnet ${ }^{1}$, H. Ezzaier ${ }^{1,2}$, A. Zubarev ${ }^{3}$ and G. Bossis ${ }^{1}$

${ }^{1}$ University of Nice Sophia Antipolis, CNRS UMR 7336, Laboratory of Condensed Matter Physics, Parc Valrose, 06108 Nice Cedex 2, France

${ }^{2}$ Laboratory of Physics of Lamellar Materials and Hybrid Nano-Materials, Faculty of Sciences of Bizerte, University of Carthage, 7021 Zarzouna, Tunisia

${ }^{3}$ Department of Mathematical Physics, Ural State University, 51, Prospekt Lenina, Ekaterinburg 620083 Russia

* Corresponding author: kuzhir@unice.fr

Keywords: phase separation; ferrofluid; magnetic colloids; magnetic separation

\begin{abstract}
In this work, we briefly review magnetic separation of ferrofluids composed of large magnetic particles (60 $\mathrm{nm}$ of the average size) possessing an induced dipole moment. Such ferrofluids exhibit field-induced phase separation at relatively low particle concentrations ( $\sim 0.8 \%$ vol.) and magnetic fields $(\sim 10 \mathrm{kA} / \mathrm{m})$. Particle aggregates appearing during the phase separation are extracted from the suspending fluid by magnetic field gradients much easier than individual nanoparticles in the absence of phase separation. Nanoparticle capture by a single magnetized microbead and by multi-collector systems (packed bed of spheres and micro-pillar array) has been studied both experimentally and theoretically. Under flow and magnetic fields, the particle capture efficiency $\Lambda$ decreases with an increasing Mason number for all considered geometries. This decrease may become stronger for aggregated magnetic particles $\left(\Lambda \propto \mathrm{Ma}^{-1.7}\right)$ than for individual ones $\left(\Lambda \propto \mathrm{Ma}^{-1}\right)$ if the shear fields are strong enough to provoke aggregate rupture. These results can be useful for development of new magneto-microfluidic immunoassays based on magnetic nanoparticles offering a much better sensitivity as compared to presently used magnetic microbeads.
\end{abstract}

\section{Introduction}

Magnetic separation is a process in which magnetically susceptible particles are extracted from a mixture using a magnetic force. It has found numerous applications in ore beneficiation industry [1], in bio-analysis for separation or detection of biological cells or molecules [2] as well as in water purification from organic or inorganic micro-pollutants [3]. The last two applications are based on adsorption of biological or pollutant molecules onto a surface of magnetic particles bearing specific chemical groups, followed by particle 
separation from the suspending fluid by a magnetic force created by an applied non-uniform magnetic field. For biomedical applications, magnetic separation is often realized in microfluidic scale allowing analysis of small volume bio-analytes and considerable reduction of high-cost chemicals [4]. In both biomedical and environmental applications, the use of nanoparticles instead microbeads is beneficial because the nanoparticles has a much larger specific area and can capture more molecules per unit volume of the suspension that will result in either a better sensitivity of biological assays or a lower consumption of nanoparticles for water purification. However until now, the broad use of nanoparticles has been limited by their strong Brownian motion making inefficient their magnetic separation.

In this work, we will review our recent investigations showing that the magnetic separation of nanoparticles of the size of about $50 \mathrm{~nm}$ can still be efficient at low-to-moderate applied magnetic fields $(5-10 \mathrm{kA} / \mathrm{m})$. In fact, the applied field could promote their phase separation manifesting through appearance of drop-like or chain-like aggregates; these aggregates have a large enough volume to become non-Brownian and they are separated from the suspending fluid much easier than individual nanoparticles. In practice, we deal with a separation of the solid phase of a ferrofluid (colloidal suspension of magnetic nanoparticles) from its liquid phase. We will start with a brief description of the field induced phase transition in ferrofluids extending existing theories [5-8] to the present case of medium sized nanoparticles with multipolar magnetic interactions [Sec. 2]. Then, separation of nanoparticles in the simplest geometry - capture by a spherical magnetizable microbead - will be considered in Sec. 3. Sections 4 and 5 will be devoted to filtration of nanoparticles on a packed bed of magnetized microbeads and to the microfluidic filtration on an ordered array of magnetized micro-pillars, respectively. The last geometry approaches the systems currently used for realization of magneto-microfluidic immunoassays with magnetic microbeads [4]. However, replacement of microbeads by much smaller nanoparticles changes the physics of the magnetic separation process, especially if nanoparticles undergo a field-induced phase transition. The conclusions and perspectives will be outlined in Sec. 6 .

\section{Field-induced phase transition}

Magnetic nanoparticles of ferrofluids exhibit dipole-dipole magnetic interactions. If the particle concentration and/or the applied magnetic field are strong enough, dipole-dipole interactions can lead to a phase separation of the ferrofluid, i.e. appearance of dense particle aggregates (concentrated phase) separated by a suspending liquid containing a small amount of nanoparticles (dilute phase). The two key parameters governing the phase separation (or 
phase transition) are the nanoparticle volume fraction $\phi$ and the dipolar coupling parameter defined as the ratio of the energy of dipole-dipole interactions between nanoparticles to the thermal energy $k_{B} T[9,10]$ :

$$
\alpha \sim \underbrace{\frac{m^{2}}{\mu_{0} k_{B} T r_{n}^{3}}}_{\substack{\text { permanent and } \\ \text { induced dipoles }}}=\underbrace{\frac{2 \pi \beta_{n}{ }^{2} \mu_{0} H_{0}{ }^{2} r_{n}{ }^{3}}{3 k_{B} T}}_{\text {induce ddipoles only }},
$$

where $m$ is the absolute value of the particle dipole moment; $\mu_{0}=4 \pi \cdot 10^{-7} \mathrm{H} / \mathrm{m}$ is the magnetic permeability of vacuum, $r_{n}$ is the nanoparticle radius; $\beta_{n}=\left(\mu_{n}-1\right) /\left(\mu_{n}+2\right)$ and $\mu_{n}$ are, respectively, the magnetic contract factor and relative magnetic permeability of nanoparticles with induced dipole moments; $H_{0}$ is the intensity of the applied field.

In the general framework of the phase transition theory, the equilibrium between the two considered phases is found by the equilibrium of nanoparticle chemical potentials, $\zeta$, and osmotic pressures, $p$, in each phase [11]:

$$
\zeta\left(\phi_{c}, \alpha\right)=\zeta\left(\phi_{d}, \alpha\right) ; \quad p\left(\phi_{c}, \alpha\right)=p\left(\phi_{d}, \alpha\right),
$$

where the subscripts " $c$ " and " $d$ " stand for the concentrated and dilute phases, respectively.

The thermodynamic functions $\zeta$ and $p$ depend on the interaction potential between nanoparticles. The existing theoretical models propose different ways to estimate magnetic and short-range non-magnetic interactions between nanoparticles that results in different expressions for $\zeta$ and $p$ and leads to different shapes of the phase diagram [5-8]. In particular case of our experiments, we deal with permanent near spherical clusters of a medium size ranging between 50 and $80 \mathrm{~nm}$ and composed of iron oxide nanoparticles of a medium size of $10 \mathrm{~nm}$. These nanoclusters can be seen as multi-domain particles without permanent dipole moment but having a relatively strong initial magnetic permeability, $\mu_{n}=10-30[12,13]$. This implies multi-polar interactions between nanoclusters at short distances between them characteristic for the concentrated phase [13]. Both from hydrodynamic and magnetic point of views, the nanoclusters behave as individual spherical particles possessing their proper hydrodynamic mobility and induced magnetic moment. Thus, their dipolar coupling parameter $\alpha$ is defined by the right-hand side of Eq. (1). For the sake of clarity the nanoclusters will be hereinafter called magnetic particles.

Implementing multi-polar and hard-sphere interactions into the model and assuming that the dilute ferrofluid phase corresponds to a disordered fluid and the concentrated phase to a face centered cubic (fcc) solid, we get appropriate expressions for the functions $\zeta$ and $p$ in both phases. Substituting these functions to Eq. (2), we get a system of two algebraic 
equations that is solved numerically with respect to the particle concentrations $\phi_{c}$ and $\phi_{d}$ at different dipolar coupling parameters $\alpha$. So obtained functions, $\phi_{c}(\alpha)$ and $\phi_{d}(\alpha)$ correspond to the binodal curves of the $\alpha-\phi$ diagram plotted in Fig. 1. These two curves separate the phase diagram into the three regions, as follows: the disordered fluid situating below the left binodal curve; the fcc-solid situating below the right binodal curve and the fluid-solid mixture occupying the space between the two binodals [13]. This mixture corresponds to drop-like aggregates dissolved in a dilute suspension of isolated magnetic particles. For our application, we are more interested by the left binodal curve, showing the lower limit of particle aggregation. At magnetic fields $H_{0}>10 \mathrm{kA} / \mathrm{m}(\alpha>2)$, the particles get aggregated at low concentrations, $\phi<8 \cdot 10^{-3}(<0.8 \%$ vol.) This is beneficial for the magnetic separation since the aggregates are easier separated from the suspending fluid than individual particles.

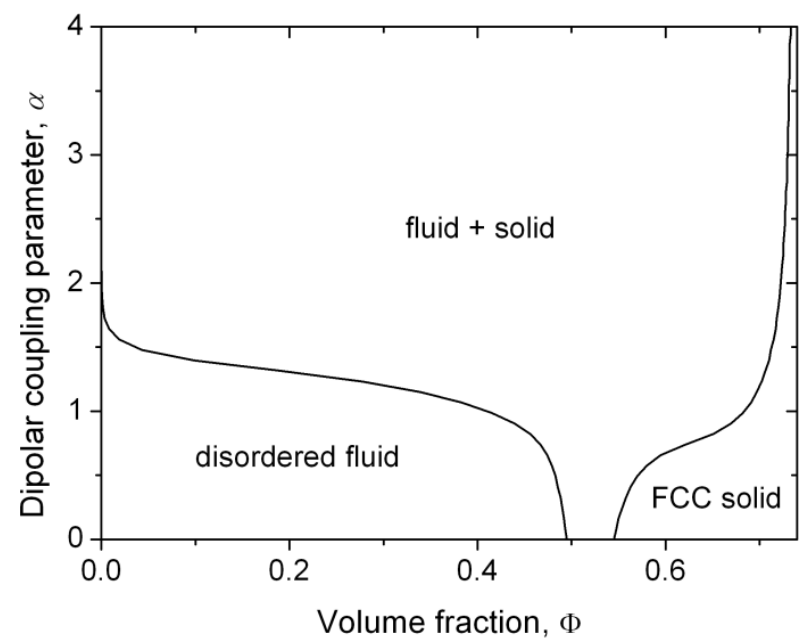

Fig.1. Phase diagram of the ferrofluid composed of magnetic particles having an induced dipole moment and a constant magnetic permeability equal to $\mu_{n}=30$ (reprinted from Magnet et al., Phys. Rev. E 89, 032310 (2014), with kind permission from American Physical Society).

\section{Separation on a single microbead}

To understand the basic mechanisms of the magnetic filtration of a phase separating ferrofluid, we start with the simplest geometry where the ferrofluid flows past a single microbead magnetized by an external magnetic field. Depending on the balance between hydrodynamic forces and magnetic forces between ferrofluid particles and micro-bead, the particles are either captured by the micro-bead or carried away from the micro-bead by the flow. During time, the captured particles build dense deposits around the micro-bead. These deposits grow with time until some steady state size when the quantity of arriving particles equilibrates the quantity of the particles eroding from the deposit surface by hydrodynamic forces. In this Section, we describe the effect of the flow and the magnetic field on the steady- 
state shape and size of the particle deposits, the deposit size being a measure of the particle capture efficiency.

The experimental cell used for visualization of particle deposits around a magnetized micro-bead is shown in Fig. 2a. The ferrofluid was composed of iron oxide particles (nanoclusters) of a medium size of $62 \mathrm{~nm}$ dispersed in water at a volume fraction $\phi_{0}=3.2 \cdot 10^{-3}$ $(0.32 \%$ vol.) and stabilized by an oleate double layer. The ferrofluid was pushed through a slit channel by a syringe pump at different imposed flow rates $Q$ corresponding to the filtration speeds, $u=Q / A$, varying in the range $1.67 \cdot 10^{-4} \leq u \leq 1.79 \cdot 10^{-3} \mathrm{~m} / \mathrm{s}$, with $A-$ the cross-section area of the channel. The flow channel was fabricated by squeezing of a silicon joint between a flat Plexiglas substrate and a microscopic glass plate. Before manufacturing of the channel, spherical nickel microparticles of a mean size ranging between 10 and $50 \mu \mathrm{m}$ were attached to the glass plate by heating at $700^{\circ} \mathrm{C}$ in an oven during two hours. The flow channel was placed in the transmitted light microscope equipped with a CMOS camera. A stationary magnetic field, longitudinal or transverse with respect to the main flow, with intensity $0 \leq H_{0} \leq 16 \mathrm{kA} / \mathrm{m}$, was applied by a pair of Helmholtz coils placed around a microscope.
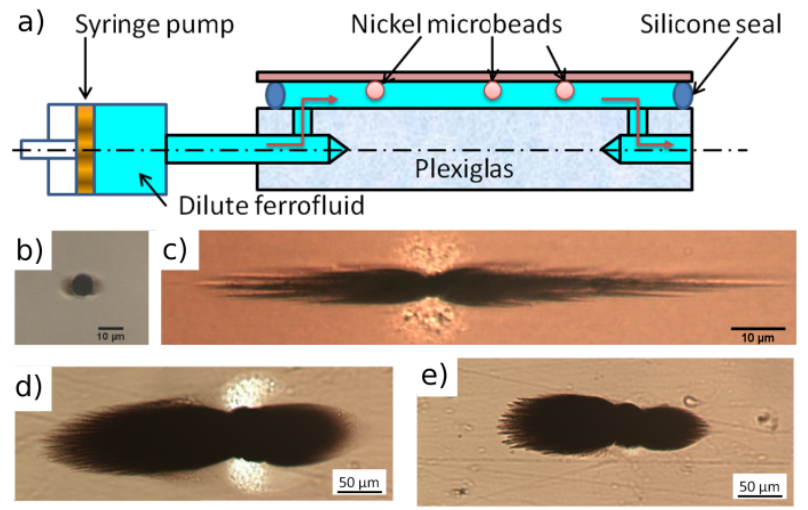

Fig.2. Sketch of the experimental setup for separation on a single microbead (a). Snapshots (b) and (c) show a nickel microbead with accumulated nanoparticles in the absence of flow and in the presence of an external uniform magnetic field of an intensity $H_{0}=8 \mathrm{kA} / \mathrm{m}$ and oriented horizontally with respect to the figure. The particle concentration in the ferrofluid is set to $\phi_{0}=0.005 \%$ vol. (b) and $0.16 \%(\mathrm{c})$. The snapshots (d) and (e) illustrate deposits of magnetic particles around the microbead in the presence a magnetic field and in the presence of the flow oriented from the left to the right of the figure, parallel to the magnetic field direction. The filtration speed $u$ is equal to $4.05 \cdot 10^{-4} \mathrm{~m} / \mathrm{s}(\mathrm{d})$ and $1.79 \cdot 10^{-3} \mathrm{~m} / \mathrm{s}(\mathrm{e})$. The snapshots (b) and (c) were taken three hours after the moment of the field application, while (d) and (e) - two hours from the moment of the flow onset.

The snapshots of the visualized steady-state particle deposits around the micro-bead are shown in Figs. $1 \mathrm{~b}$ and $1 \mathrm{c}$ in the absence of flow at the same applied magnetic field $H_{0}=8$ $\mathrm{kA} / \mathrm{m}$ but at two very different initial particle concentrations: $\phi_{0}=0.005 \% \mathrm{vol}$. and $0.16 \% \mathrm{vol}$. At the lowest concentration, there is no apparently any phase separation in the ferrofluid; one can only see a transparent halo around the microbead corresponding to Boltzmann distribution 
of particle concentration [Fig. 1b]. At the highest concentration, the ferrofluid undergoes the phase separation: long string-like particle aggregates were observed in the ferrofluid bulk; these aggregates progressively migrated to the microbead and formed dense and long deposits extended along the applied magnetic field [Fig. 1c]. The content of the deposits can be seen as a concentrated ferrofluid phase, while the surrounding transparent medium corresponds to the dilute phase. These two snapshots clearly show that the phase separation enhances significantly the particle capture efficiency. Under flow, the size and shape of the particle deposits evolve. The snapshots $1 \mathrm{~d}$ and $1 \mathrm{e}$ show that their size decreases with the filtration speed because the hydrodynamic forces, eroding the particles from the deposit surface, become stronger with increasing speed. Asymmetry of the front (facing the flow) and back deposits likely comes from the asymmetry of the velocity profile resulting in difference in hydrodynamic forces acting on the front and on the back deposits. Finally, the spikes on the deposit surface (both in the presence and in the absence of field) can be explained by a surface instability appearing because of negative surface energy in the vicinity of the deposit extremities where the magnetic field is nearly orthogonal to the deposit surface $[14,15]$.

Let us now estimate the deposit's size and shape and compare them to the ones observed in experiments. Analysis shows that in the considered range of experimental parameters, the steady-state deposit shape is not significantly influenced by the flow. It can be determined from the continuity of the normal component of the stress tensor on the surface excluding the viscous term. This gives us the following differential equation for the deposit surface described by the geometric locus $[R(\theta), \theta]$ in the polar coordinate system [12]:

$$
h_{0}{ }^{2}-h^{2}=\chi \frac{\left(h_{r}-h_{\theta} R^{\prime} / R\right)^{2}}{1+\left(R^{\prime} / R\right)^{2}},
$$

where $R^{\prime}=d R / d \theta ; \chi \approx 9$ is the deposit magnetic susceptibility; $h_{r}=\left(1+2 \beta_{m} / R^{3}\right) \cos \theta$ and $h_{\theta}=-\left(1-\beta_{m} / R^{3}\right) \sin \theta$ are respectively, the radial and the polar components of the magnetic field intensity, both normalized by the applied field $H_{0} ; \beta_{m} \approx 1$ is the magnetic contrast factor of the microbead; $h=\sqrt{h_{r}^{2}+h_{\theta}{ }^{2}}$ is the absolute value of the normalized magnetic field at a given point $(R, \theta)$ of the deposit surface and $h_{0} \approx 3 \cos \theta_{0}$ stands for the value of $h$ at the point ( $R=1$ and $\theta=\theta_{0}$ ) where the deposit surface joins the microbead; the angle $\theta_{0}$ [cf. Fig. 3] is called the anchoring angle. The ordinary differential equation (3) is solved numerically at the initial condition $R\left(\theta_{0}\right)=1$. 
In the present model, the anchoring angle defines the deposit volume. In the absence of flow, we measure the angle $\theta_{0} \approx 69 \mathrm{deg}$, while in the presence of flow the balance of particle fluxes on the deposit surface allows us to find approximate relationships (not given here for brevity) between the anchoring angle (and, consequently, the deposit size) and the Mason number describing the ratio of hydrodynamic-to-magnetic forces exerted on magnetic particles [13]:

$$
M a=\frac{\eta u}{\mu_{0} \beta_{m} \beta_{n} H_{0}^{2} r_{n}},
$$

where $\eta$ is the viscosity of the suspending liquid of the ferrofluid (water in our case).

The calculated deposit shape is compared to the experimentally observed one on Fig. 3 for the longitudinal magnetic field and two different values of the Mason numbers equal to 0.08 and 0.35 . Our model reproduces qualitatively the elongated shape of the deposits. It correctly predicts an asymmetry of the front and rear deposits in the longitudinal field, as observed in experiments. Because of the simplifying input hypotheses, the model is unable to reproduce the surface instability manifested through appearance of conical spikes on the deposit surface.
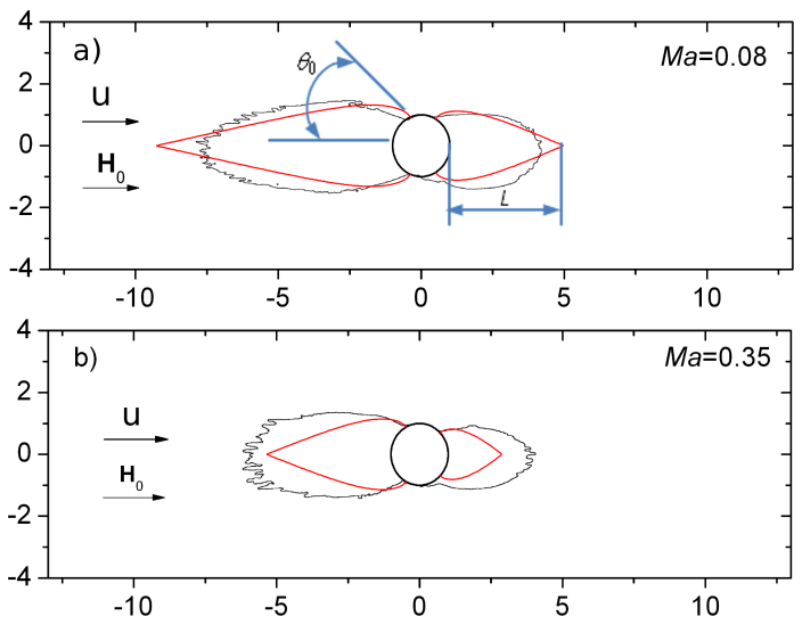

Fig. 3. Comparison between the calculated (red solid line) and the experimental (black wavy line) shapes of the particle deposits attached to the microbead in the presence of an external magnetic field and flow both oriented horizontally with respect to the figure. The Mason number Ma is set to 0.08 (a) and 0.35 (b) (reprinted from Magnet et al., Phys. Rev. E 89, 032310 (2014), with kind permission from American Physical Society).

Theoretical and experimental dependencies of the steady state deposit length $L$ (distance from the micro-bead surface to the deposit extremity, cf. Fig. 3) on the Mason number are shown on Fig. 4 for both longitudinal and transverse magnetic fields. In the range of Mason numbers, $0.06<\mathrm{Ma}<0.5$, the length of the front deposit monotonically decreases with the Mason number, which is explained by increasing hydrodynamic forces washing away the magnetic particles from the deposit surface. On the contrary, the length of the back deposit 
is much less affected by the flow and remains almost constant within the considered range of Mason numbers. Our model qualitatively reproduces the decreasing trend for the front deposit and gives a quantitative correspondence with experiments for the longitudinal magnetic field with maximum of $35 \%$ of discrepancy. The experimental deposit length in the transverse field appears to be somewhat smaller than that of the front deposit in the longitudinal field likely because stronger hydrodynamic forces are exerted to the deposits oriented perpendicularly to the flow. Finally, our theory reveals that the magnetic interactions between magnetic particles dominate over Brownian motion at the dipolar coupling parameters $\alpha \geq 2$ and the particle magnetic permeability $\mu_{n} \geq 30$. At these conditions, the Mason number is the main parameter governing the efficiency of the filtration of a phase separating ferrofluid on a single magnetized microbead.

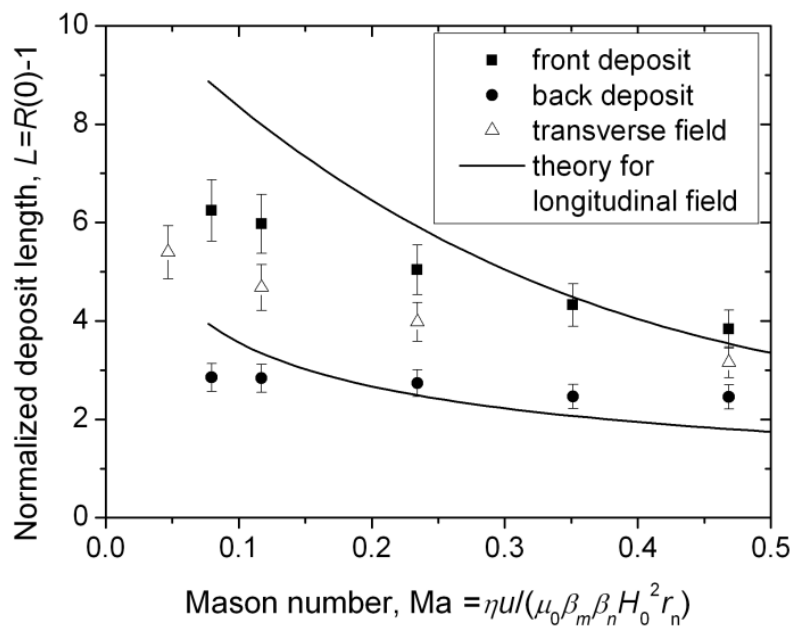

Fig.4. Theoretical and experimental Mason number dependencies of the deposit length normalized by the microbead radius. The upper solid line stands for the theoretical prediction for the front deposit in the longitudinal field. The lower solid line stands for the prediction for the back deposit in the longitudinal field (reprinted from Magnet et al., Phys. Rev. E 89, 032310 (2014), with kind permission from American Physical Society).

\section{Separation on a packed bed of microbeads}

In a real magnetic separator, magnetic particles are separated from the suspending liquid not by a single micro-bead but by numerous more or less closely spaced collectors magnetized by an external magnetic field. The particle capture on a given collector is affected by neighboring collectors because they modify the velocity and the magnetic field around a given collector. A packed bed of magnetizable micro-beds is one of possible configurations of the magnetic separation system. In this Section, we study magnetic filtration through such a system and seek for the Mason number effect on the capture efficiency.

In experiments, strongly diluted ferrofluid (solid phase concentration $\phi_{0}=1.6 \cdot 10^{-5}$ $(0.0016 \%$ vol.) is subjected to a recirculation flow in a closed loop circuit containing a 
magnetic filter whose ends are connected with each other by flexible tubes, as depicted in Fig. 5a. A peristaltic pump is included into the circuit and ensures the flow with a constant flow rate $Q$ corresponding to the filtration speeds, $u=Q / A$, inside the filter, ranging from 0.03 to $0.12 \mathrm{~m} / \mathrm{s}$. The filter itself is a cylindrical column, filled with nickel microbeads of a mean diameter $2 r_{m}=45 \pm 5 \mu \mathrm{m}$ at a volume fraction of about $c \approx 0.3$. A solenoid is placed around the filter and generates a magnetic field parallel to the flow direction and having an intensity up to $H_{0}=32 \mathrm{kA} / \mathrm{m}$. Once the field is applied and the flow is started, the filter becomes to capture the magnetic particles such that their concentration $\phi$ outside the filter (in the tubes) starts to decrease with the time. The temporal evolution of the concentration $\phi(t)$ is measured by a turbidimeter as function of the filtration speed $u$ and of the applied field $H_{0}$.
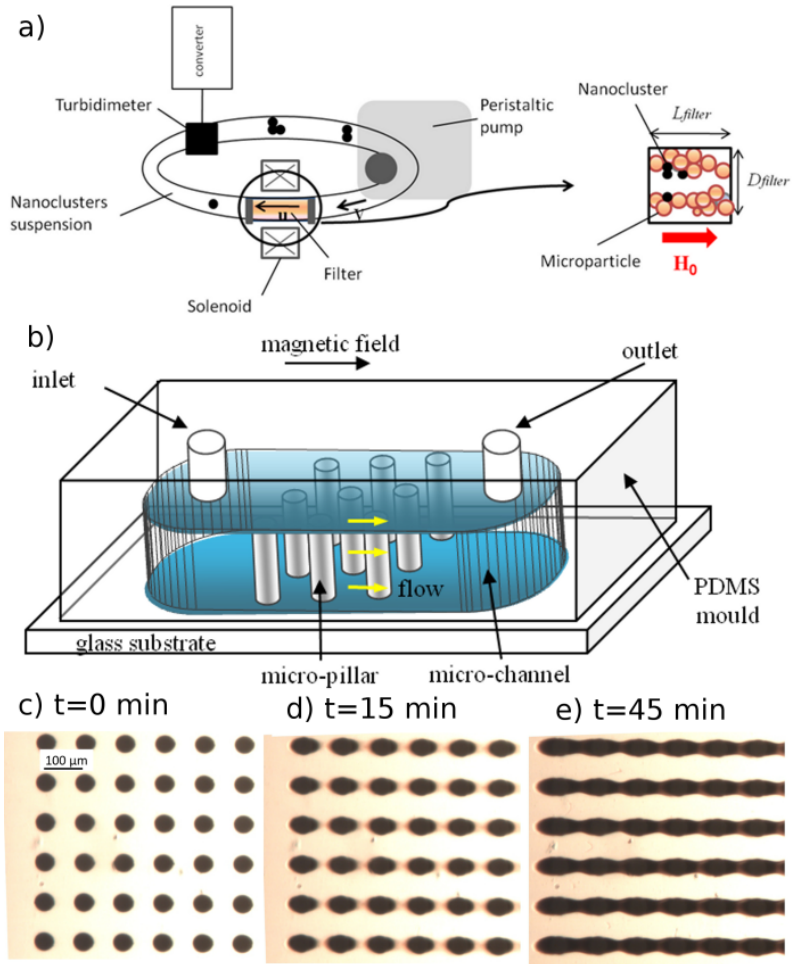

Fig. 5. Sketch of the experimental setups used for the filtration through a packed bed of microbeads (a) and through a microfluidic channel with a micro-pillar array (b). Optical microscopy images (c) - (e) show the evolution of the particle deposits around micro-pillars with time at the magnetic field intensity $H_{0}=6.05 \mathrm{kA} / \mathrm{m}$, filtration speed $u=1.88 \cdot 10^{-3} \mathrm{~m} / \mathrm{s}$ and initial particle concentration in the ferrofluid $\phi_{0}=0.32 \% \mathrm{vol}$. The snapshot (c) at $t=0$ corresponds to the micro-pillars free of magnetic particles.

The measured $\phi(t)$ curves are then fitted to the phenomenological filtration law derived from the solution of the filtration equation [16] taking the following form for the closed loop geometry at low initial particle concentrations ${ }^{1} \quad \phi_{0}<<1$ and at low-to-moderate capture efficiencies $\Lambda<1$ [17]:

\footnotetext{
${ }^{1}$ Approximate expression (5) is obtained by setting $\gamma_{2}$ to unity in Eq. (3) of the original paper [17]
} 


$$
\phi \approx \phi_{0} \exp \left(-V \Lambda \tau / V_{\Sigma}\right)
$$

where $\tau=u t / L$ is the dimensionless time; $L$ and $V$ are the filter length and volume, respectively; $V_{\Sigma}$ is the total volume of fluid phase in the closed-loop circuit. The single adjustable parameter of this fit is the capture efficiency $\Lambda$ - a phenomenological coefficient defined through the ratio of particle concentrations at the filter inlet $\left(\phi_{\text {in }}\right)$ and outlet $\left(\phi_{\text {out }}\right)$ at the beginning of the filtration: $\Lambda=\ln \left(\phi_{\text {in }} / \phi_{\text {out }}\right)$. This coefficient is finally analyzed as a function of Mason number. From the theoretical point of view, the capture efficiency can be related to the Mason number by calculating magnetic particle flux arriving to one of the microbeads situating inside the packed bed. Analytical solution of this problem is still possible in the frame of the concentric-sphere model of the packed bed, in the limit Ma $r_{m} / r_{n}>>1$ and under assumption of negligible Brownian motion. Furthermore, we consider magnetic beads with a high magnetic permeability $\left(\beta_{m} \approx 1\right)$ and assume that all the particle aggregates are destroyed in the considered range of Mason numbers. This gives us the following expression for $\Lambda$, valid at $\mathrm{Ma}_{m} / r_{n}>3 \cdot 10^{3}$ or at $\mathrm{Ma}>3$ [17]:

$$
\Lambda \approx 12 \frac{c}{(1-c)^{2}} \frac{L r_{n}}{r_{m}^{2}} \mathrm{Ma}^{-1} .
$$

The effect of the neighbourhood of the microbeads of the packed bed on the capture efficiency of magnetic particles is described by the term $c /(1-c)^{2}$ showing stronger than linear increase of the efficiency with the volume fraction $c$ of the microbeads. The theoretical and experimental (obtained by fitting experimental $\phi(t)$-curves to Eq. (5)) Mason number dependencies are shown on Fig. 6. Both the theory (dashed curve) and experiments (open triangles) show a decreasing Mason number dependency of the particle capture efficiency. Such behaviour is easily explained by increasing hydrodynamic forces transporting the particles through the filter and hindering their capture by the microbeads. Quantitatively, our model [Eq. (6), dashed curve] allows a correct prediction of the capture efficiency only at Mason numbers Ma $>4$, while it strongly underestimates the value of $\Lambda$ at lower Mason numbers. The discrepancy rises likely because of the field-induced phase separation occurring at $\mathrm{Ma} \leq 1$ and ignored in the present model. In fact, the experimental point at $\mathrm{Ma} \approx 1$ (encircled point on Fig.6) corresponds to the value of the dipolar coupling parameter, $\alpha \approx 20$, at which the phase separation is expected to occur for the considered particle concentration $\phi_{0}=1.6 \cdot 10^{-5}$, while the data for $\mathrm{Ma}>4$ correspond to $\alpha \approx 5$, for which the particles do not undergo phase 
separation. The effect of a possible aggregation of magnetic particles on their capture efficiency will be analyzed in the next Section 5 for a simpler and more important (for biomedical applications) microfluidic magnetic separation system.

\section{Microfluidic separation on an ordered array of micro-pillars}

For applications to bioassays, it is often beneficial to realize ordered and more or less sparse arrays of magnetic collectors and reduce the size of the separator to microfluidic scale. In this Section we will describe magnetic filtration using one of such systems and will characterize the capture efficiency paying special attention to field-induced particle chaining.

To this end, we fabricated a nickel micro-pillar array on a glass substrate by electroplating and soft photolithography, according to a general procedure described by Deng et al. [18]. A polydimethylsiloxane (PDMS) cover was glued to the glass substrate bearing the nickel micro-pillars using oxygen plasma. Aluminum needles were introduced to the PDMS cover at both its extremities allowing the channel inlet and outlet. The resulting microfluidic channel is schematically presented in Fig. $5 \mathrm{~b}$ and an optical microscopy image of the micropillar array is shown in Fig. 5c. In experiments, we used a square 10x10 array with micropillar diameter of $2 r_{m}=50 \mu \mathrm{m}$ and expected height of $h=50 \mu \mathrm{m}$ and a distance between micropillar axes of $\Delta=100 \mu \mathrm{m}$. The fraction of the filter volume occupied by the pillars was $c \approx 0.2$. The diluted ferrofluid (composed of nanoclusters of a mean diameter $2 r_{n} \approx 84 \mathrm{~nm}$ dispersed in water at volume fraction of $\phi_{0}=3 \cdot 2 \cdot 10^{-3}$ ) was pushed through the microfluidic channel by a syringe pump at the flow rates $Q$ corresponding to the range of filtration speeds $u=Q / A=3.4 \times 10^{-5}-3.4 \times 10^{-3} \mathrm{~m} / \mathrm{s}$. Accumulation of deposits of magnetic particles around the micro-pillars in the presence of flow and of an external magnetic field oriented along the flow was visualized and recorded using the equipment described in Section 3. A few snapshots showing progressive growth of the deposits are shown in Figs. 5c-e. At certain conditions, oval shape of the deposits at short times from the beginning of the capture [Fig. 5d] evolves to a continuous wavy pattern when the deposits join together all the micro-pillars along the direction of the applied field [Fig. 5e]. Image processing allowed us to follow the evolution of the surface $S$ occupied by nanoparticle deposits with time. This surface was then normalized by the total area of the filter $S_{\text {tot }}$. The time dependency of the relative deposit area $s=S / S_{\text {tot }}$ was then fitted to the $s(t)$-dependency obtained by integration of the phenomenological filtration equations [16] under assumption of relatively low capture efficiency $\Lambda<1$ [10]:

$$
s(\tau) \approx s_{m}\left[1-\exp \left(-\phi_{0} \Lambda \tau /\left(\Phi s_{m}\right)\right)\right]
$$


where $s_{m}$ is the maximal relative deposit area when the filter is saturated with captured particles, $\Phi \approx 0.74$ is estimated internal volume fraction of deposits; the dimensionless time $\tau$ and the capture efficiency $\Lambda$ are introduced in the same way as for filtration through a packed bed [see Sec. 4]. The capture efficiency, $\Lambda$, was used as an adjustable parameter of this fit, and its value was analyzed as a function of Mason number.

The theoretical dependency $\Lambda(\mathrm{Ma})$ was obtained in the limit $\mathrm{Ma} r_{m} / r_{n}>>1$ in a similar way as in Sec. 4, i.e. by integration of the particle flux on the surface of the micro-pillar but the model was extended to the case of field-induced aggregation of magnetic particles that is expected for larger particle concentrations used for the micro-pillar geometry $\left(\phi_{0}=3.2 \cdot 10^{-3} \mathrm{vs}\right.$ $1.6 \cdot 10^{-5}$ for filtration through a packed bed). We supposed that magnetic particles assembled in straight chain-like aggregates with the number of particles per aggregate denoted by $N$. The aggregate length (or, equivalently, $N$ ) was defined by the balance of an average hydrodynamic tensile force rupturing the chain and an average attractive magnetic force between particles inside the chain. Such reasoning was borrowed from the chain model of electrorheology [19] and allowed the following scaling for the particle number per chain: $N \propto \mathrm{Ma}^{-1 / 2}$. Incorporating this issue to the capture efficiency, we obtain the following Mason number law, approximately valid for relatively short chains $(N \leq 8)$ and for the Mason numbers corresponding to the limit $\mathrm{Ma} r_{m} / r_{n}>>1[10]$ :

$$
\Lambda \approx \frac{8}{3} \frac{C}{(1-c)^{2}} \frac{L r_{n}}{r_{m}^{2}}\left(N^{7 / 5} \mathrm{Ma}^{-1}\right) \propto \mathrm{Ma}^{-1.7}
$$

The experimental Mason number dependencies of the capture efficiency (obtained by fitting the $s(t)$ curves to Eq. (7)) were fitted to the theoretical law (8) and are shown in Fig. 6 by solid triangles and open squares for two values of the applied magnetic field, $H_{0}=6.05$ and $10.9 \mathrm{kA} / \mathrm{m}$. The data for both fields (corresponding to the dipolar coupling parameter $\alpha=1.4$ and 4.5) collapse onto a straight line in a log-log scale, corresponding to the theoretical scaling law (8) (solid line on Fig. 6). The observed scaling behavior, $\Lambda \propto \mathrm{Ma}^{-1.7}$, qualitatively supports our hypothesis on a strong decrease of the capture efficiency with Mason number because of destruction of particle aggregates (chains) with increasing filtration speeds. Comparing Mason number effects for the micro-pillar and packed bed geometries, we notice on Fig. 6 a less pronounced decrease of the capture efficiency for the packed bed $\left(\Lambda \propto \mathrm{Ma}^{-1}\right)$ than for the micro-pillars $\left(\Lambda \propto \mathrm{Ma}^{-1.7}\right)$ related to the fact that there is no phase separation or particle aggregation in the first case (at very small particle concentration, $\phi_{0}=1.6 \cdot 10^{-5}$, except 
for the encircled point), while particle aggregation is expected in the second case. Finally, $\Lambda(\mathrm{Ma})$-curve for the packed bed (dashed curve on Fig.6) lies above the $\Lambda(\mathrm{Ma})$-curve for the micro-pillar geometry (solid line). This could be explained by geometrical differences between both configurations, such as higher collector volume fraction $c$ and higher length $L$ of the packed bed filter.

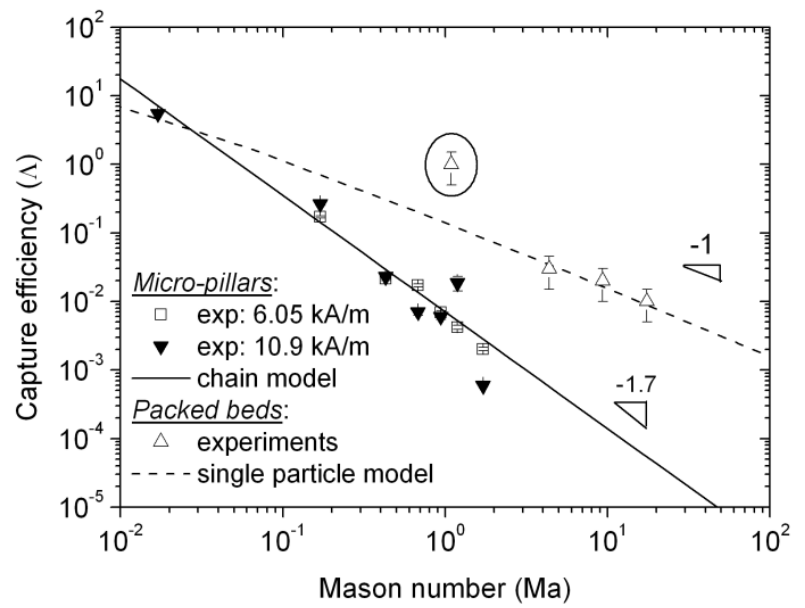

Fig.6. Experimental and theoretical dependencies of the capture efficiency on Mason number for a packed bed filter and a microfluidic filter equipped with a micro-pillar array. The encircled point at $\mathrm{Ma} \approx 1$ corresponds to a phase separating ferrofluid, while the three remaining points of the same curve correspond to individual magnetic particles. The dashed curve corresponding to the single particle model was obtained for a broad range of Mason numbers by numerical particle trajectory analysis giving $\Lambda \propto \mathrm{Ma}^{-0.83}$ at $0.03<\mathrm{Ma}<3$ and reproducing the analytical result $\Lambda \propto \mathrm{Ma}^{-1}$ [Eq. (6)] at Ma>3. Notice that a slightly different definition of $\mathrm{Ma}$ (using another length-scale) was used in the original papers [10,17] leading to expressions for $\Lambda$ different from Eqs. (6) and (8) of the present paper.

\section{Conclusions and perspectives}

In this work, we have briefly reviewed magnetic separation of ferrofluids composed of large magnetic particles (spherical nanoclusters of a size of the order of $60 \mathrm{~nm}$ ) possessing an induced dipole moment. In the presence of an applied uniform magnetic field, these ferrofluids undergo a fluid-solid phase transition fully governed by the particle volume fraction $\phi$ and the dipolar coupling parameter $\alpha$. At moderate magnetic fields, $H_{0} \sim 10 \mathrm{kA} / \mathrm{m}$ $(\alpha \sim 2)$, the phase transition occurs at relatively low particle volume fraction ( $\phi \sim 0.8 \%$ vol.) and is accompanied by appearance of particle drop-like or chain-like aggregates. Such fieldinduce aggregation strongly enhances the capture efficiency of magnetic particles both by single and multi-collector systems. At favorable conditions, the aggregates become large enough to be non-Brownian and their capture by magnetized collectors depends only on the ratio of hydrodynamic-to-magnetic forces - the Mason number. The capture efficiency on a single microbead is characterized by the steady-state size of magnetic particle deposits around the microbead while it is defined through the ratio of the particle concentration at the inlet and 
outlet for filtration through multi-collector systems (packed bed or micro-pillar array). In all considered geometries, the capture efficiency decreases with an increasing Mason number because of hydrodynamic forces washing away magnetic particles from the collectors. This decrease may become stronger for aggregated magnetic particles $\left(\Lambda \propto \mathrm{Ma}^{-1.7}\right)$ than for individual ones $\left(\Lambda \propto \mathrm{Ma}^{-1}\right)$ if the shear fields are strong enough to provoke aggregate rupture, as is expected for the microfluidic filtration through the micro-pillar array. However, the filter geometry could be optimized to promote particle aggregation well before they arrive to the collectors and to avoid the aggregate rupture in the vicinity of the collectors. This imposes combined engineering and fundamental studies of the kinetics of particle aggregation and of aggregate behaviors in the microfiltration devices. These future studies should allow development of new magneto-microfluidic immunoassays based on magnetic nanoparticles offering a much better sensitivity as compared to presently used magnetic microbeads.

\section{Acknowledgements}

We are grateful to master students Y. Bourras, S. Nave, M. Acouala and G. Orlandi for experimental measurements. This work has been partially supported by the French RENATECH network and its FEMTO-ST technological facility, by the PEPS-INSIS project of CNRS and by the Russian Scientific Foundation (project $n^{\circ} 14-19-00989$ ). One of the authors (H.E.) acknowledges FSB through its PhD collaboration fellowship.

\section{References}

[1] J. Svoboda, Magnetic Techniques for the Treatment of Materials, Kluwer Academic, Dordrecht, 2004

[2] M. Zborowski and J.J. Chalmers, Magnetic cell separation, Elsevier, Amsterdam, 2008

[3] R. D. Ambashta, and M. Sillanpää, Water purification using magnetic assistance: A review, J. Hazardous Materials, 180 (2010) 38-49.

[4] M. A.M. Gijs, F. Lacharme and U. Lehmann, Microfluidic Applications of Magnetic Particles for Biological Analysis and Catalysis, Chem. Rev. 110 (2010) 1518-1563.

[5] A. O. Tsebers and M.M. Maiorov, Magnetostatic instabilities in-plane layers of magnetizable liquids, Magnitnaya Gidrodinamika 18, N² (1982) 42; A. O. Tsebers Magnetohydrodynamics 18, No.2, (1982) 137

[6] Y.A. Buyevich and A.O. Ivanov, Equilibrium properties of ferrocolloids. Physica A,190 (1992) 276-294

[7] J. Liu, E. M. Lawrence, A. Wu, M. L. Ivey, G. A. Flores, K. Javier, J. Bibette, and J.Richard, Field-Induced Structures in Ferrofluid Emulsions, Phys. Rev. Lett. 74 (1995) 2828-2831

[8] A.Y. Zubarev and L.Y. Iskakova, Theory of structural transformations in ferrofluids: Chains and - gas-liquid phase transitions. Physical Review E, 65 (2002) 061406.

[9] R.E. Rosensweig, Ferrohydrodynamics, Cambridge University Press, Cambridge - New York (1985)

[10] G. Orlandi, P. Kuzhir, Y. Izmaylov, J. Alves Marins, H. Ezzaier, L. Robert, F. Doutre, X.Noblin, C. Lomenech, G.Bossis, A. Meunier, G. Sandoz and A. Zubarev, Microfluidic separation of magnetic nanoparticles on an ordered array of magnetized micro-pillars, Phys. Rev. E 93, 062604 (2016)

[11] L.D. Landau, and E.M. Lifshitz, Statistical Physics, Vol. 1, (3rd Edition), Pergamon, Oxford, 1980

[12] C. Magnet, P. Kuzhir, G. Bossis, A. Meunier, L. Suloeva, and A. Zubarev, Haloing in bimodal magnetic colloids: the role of field induced phase separation Phys. Rev. E 86 (2012) 011404. 
[13] C. Magnet, P. Kuzhir, G. Bossis, A. Meunier, S. Nave, A. Zubarev, C. Lomenech and V. Bashtovoi, Behavior of nanoparticle clouds around a magnetized microsphere under magnetic and flow fields, Phys. Rev. E 89 (2014) 032310.

[14] A. E. Lobkovsky, and T.C. Halsey. Surface energy anisotropy for dipolar lattices. J. Chem. Phys. 103 (1995) 3737-3741.

[15] J.H.E Promislow, and A.P. Gast, Low-energy suspension structure of a magnetorheological fluid. Phys. Rev. E, 56 (1997) 642-651

[16] C. Tien, and B. V. Ramarao, Granular Filtration of Aerosols and Hydrosols, Elsevier Science \& Technology Books, New York, 2007.

[17] C. Magnet, M. Akouala, P. Kuzhir, G. Bossis, A. Zubarev, and N.M. Wereley, Closed-loop magnetic separation of nanoparticles on a packed bed of spheres J. Appl. Phys. 117 (2015) 17C719

[18] T. Deng, M. Prentiss, and G. M. Whitesides, Fabrication of magnetic microfiltration systems using soft lithography Appl. Phys. Lett. 80 (2002) 461-463

[19] J. E. Martin and R. A. Anderson, Chain model of electrorheology, J. Chem. Phys. 104, (1996) 4814-4827. 